\title{
LOCAL FEATURE EXTRACTION IN THE NEAR INFRA-RED DOMAIN FOR WILDLIFE MAMMALS TRACKING PURPOSE
}

Tibor TRNOVSZKÝ1,*, Róbert HUDEC²

${ }^{1}$ Department of Telecommunications and Multimedia, Faculty of Electrical Engineering, University of Žilina, Univerzitná 8215/1, 01026 Žilina, Slovakia.

2 Department of Telecommunications and Multimedia, Faculty of Electrical Engineering, University of Žilina, Univerzitná 8215/1, 01026 Žilina, Slovakia.

corresponding author: tibor.trnovszky@fel.uniza.sk.

\begin{abstract}
Nowadays, research in area of intelligent transportation system is focused on analysis of traffic flow by computer vision techniques. Keypoint analysis represents detection, modelling and recognition of objects in traffic flow and object tracking as well. The main goal of this paper is to propose new approach to detect and modelling 3D objects that move on road surface. At the beginning, basic methods of object recognition and modelling of $3 \mathrm{D}$ object are shortly described. The modified algorithm based on background subtraction and creation of 3D model by depth map is proposed. Finally, the results of developed algorithm are depicted through the last part of this paper.
\end{abstract}

\section{Keywords:}

Intelligent transportation system; Animal detection; Object recognition; Infrared domain; Local feature extraction.

\section{Introduction}

In Slovak Republic, there were more than 8500 car collisions with animals for last eight years. Animal collision with car was the second most common reason for car accident until 2010. The first common reason was driver's inattention. In the Table 1 is shown number of all car accidents and caranimal accidents for last eight years [1]. Many car-animal accidents are not recorded, because driver did not inform the police or hunting association and just escape from the accident place. Thus, in real life this number is bigger. Most of these collisions are mortal for animals and create unwanted decreasing of wild animals in our country and make a lot of material damage and financial loss.

Tab. 1: Car accidents in Slovak Republic for last 8 years.

\begin{tabular}{|c|c|c|c|c|}
\hline Year & $\begin{array}{c}\text { Total } \\
\text { accidents }\end{array}$ & $\begin{array}{c}\text { Animal } \\
\text { accidents }\end{array}$ & $\%$ & Kind of accident \\
\hline 2007 & 61071 & 2920 & 4,8 & 2. place \\
\hline 2008 & 59008 & 3049 & 5,2 & 2. place \\
\hline 2009 & 25989 & 991 & 3,8 & 2. place \\
\hline 2010 & 21611 & 723 & 3,34 & 2. place \\
\hline 2011 & 15001 & 244 & 1,62 & 4. place \\
\hline 2012 & 13495 & 255 & 1,89 & 5. place \\
\hline 2013 & 13586 & 197 & 1,45 & 4. place \\
\hline 2014 & 13287 & 194 & 1,46 & 4. place \\
\hline
\end{tabular}

Camera monitoring of wild animals in forests or near roads could help to gather a lot of information about amount of wild animals in monitored area, make a recognition of animals, record their movements, habits and find out seasons and time for animal migration and make preventive measures which could be used for migration corridor creation. This information can be used for planning and building roads to avoid places with a lot of animal movements and make prevention for car-animal collision accident and automatically inform police, hospital or hunting association, and reduce road construction cost and also find the best place for building barriers or wildlife crossing such 
as ecoducts and underpasses. There are a lot of advantages to start monitoring and recognizing wild animals.

Therefore, we are trying to develop automatic recognition system for mammal tracking purpose in near infrared domain which will be used for mammal recognition during the night time. One of the most important steps is local feature extraction which will be used in training process and also for testing process. Thus, comparison between some methods was realized.

\section{Infrared domain}

There are some systems for animal recognition in daylight. However, the most of wild animals are primary active in night. Therefore monitoring in night is needed. This task can be realized by using cameras in infrared spectrum, see Figure 1. Infrared spectrum is part of light that human cannot see, because longer wavelength light has lower energy and it is too low for human eyes to see it. Human eye can see only light between $380-700 \mathrm{~nm}$ wavelength and it is called visible light. Infrared domain is between $700 \mathrm{~nm}$ to $1 \mathrm{~mm}$ wavelength. With special sensors and cameras, it is possible to detect and convert this part of light into electrical impulses and construct image from it. Infrared light is divided into three subparts based on ISO 20473. First is NIR (Near Infrared Light) between $780 \mathrm{~nm}$ to $3000 \mathrm{~nm}$ wavelength. Second is MIR (Medium Infrared Light) between 3000 to $50000 \mathrm{~nm}$ and the third is FIR (Far Infrared Light) up to $1 \mathrm{~mm}$ wavelength. The key difference between NIR and MIR/FIR is that NIR is used as illumination and then reflected light from objects is captured. Thus lighter areas are areas closer to light source or have bigger reflection and darker areas are farther from light source or have smaller reflection. The MIR and FIR capture directly emitted light from objects as thermal radiation where lighter areas are hotter and darker ones are colder, it is called thermo-vision [2][3].

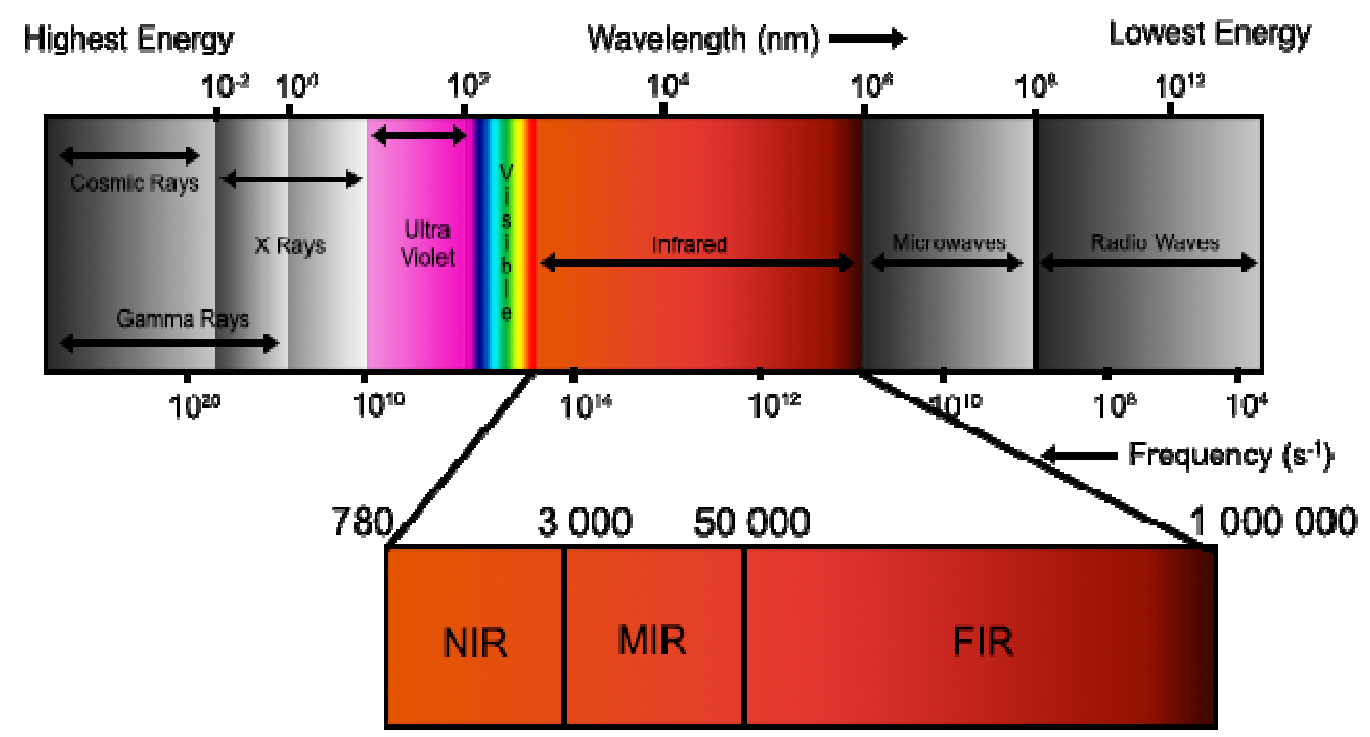

Fig. 1: Infrared spectrum.

On the Figure 2, there is a comparison of captured images from thermo-vision camera (left) and from trail camera with NIR illumination (right). As you can see, there are some big differences between images. Thermo-vision image provide very good contrast between background and foreground (animal). Most of intelligent animal recognition systems in night are based on thermo-vision cameras which have smaller resolution but they are very expensive. Quality of near infrared image depends on amount of emitted NIR light from light source. Therefore contrast between background and foreground is worse. We will try to make our algorithm usable also on low cost cameras for better implementation. Therefore, using near infrared cameras could give us good enough quality of video data with high resolution and good FPS rate for animal detection and recognition in real-time. Also based on study [4], animals should not be able to notice or see infrared light. Due to low energy of IR light animals cannot visualize stimulation in infrared light domain. Therefore, whole process of video capturing does not disturb animals. 


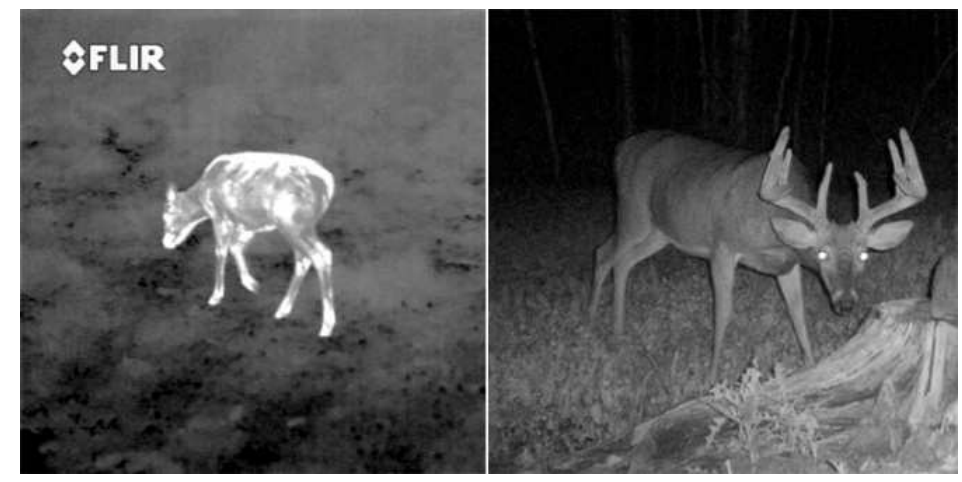

Fig. 2: Comparison of captured animal with: thermo-vision camera FLIR (left), trail camera with NIR illumination (right).

\section{System overview}

We will use our existing system ASFAR [5] and we will extend this system with NIR camera and also we will add new block for object detection and feature extraction in NIR domain mainly used for night capturing and it is illustrated on Figure 3 . This extension is highlighted in red area. For our purpose, we use security outdoor camera with night vision Axis Q1765-LE. It has four infrared LED diodes for scene illumination. Each diode emits light around $850 \mathrm{~nm}$ wavelength peak. It enables to see the objects up to $40 \mathrm{~m}$ distance during the night for capturing enough good quality video stream. Switching between daytime and nighttime mode can be automatically controlled by the camera. Based on used mode, the system will be switched between block for detection and feature extraction during the daytime and during the nighttime. Animal classification during the daytime has already been developed for ASFAR system. Now, the extension in IR object-based classification will be developed. First, we need find the best local feature extraction methods for near infrared domain video sequences. When object detection and recognition will be done, object tracking algorithm will be used.

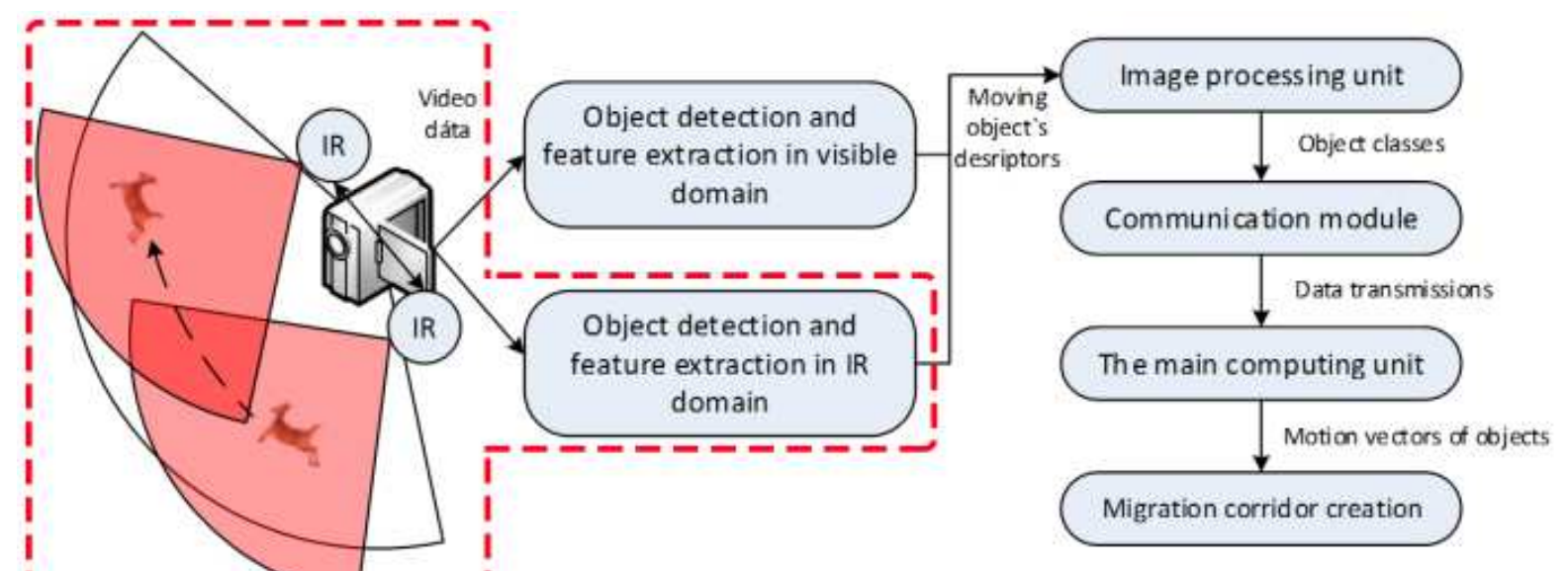

Fig. 3: Extension of ASFAR system.

\section{Local feature extraction methods}

The process of local feature extraction can be divided into two steps. First is feature detection and the second step is feature description. There are a lot of methods for local feature extraction some of them is only for detection or only for extraction and there are also methods which have both detector and descriptor. Some of the most famous methods is SIFT [6], SURF [7], BRIEF [8], ORB [9], BRISK [10], FREAK [11]. 


\subsection{SIFT}

The SIFT algorithm was published by Lowe (2004) and it includes both a keypoint detector and descriptor. Keypoint detection is done by building a scale-space representation of the original image. This is achieved by repeatedly convolving the image with a Gaussian function. A Difference of Gaussian (DoG) approach combined with interpolation over the scale-space leads to the locations of stable keypoints in that scale-space representation of the image. After that localization, each keypoint is assigned an orientation, which leads to the desired rotation invariance. The keypoint descriptors are calculated from the local gradient orientation and magnitudes in a certain neighbourhood around the identified keypoint. The gradient orientations and magnitudes are combined in a histogram representation, from which the descriptor is formed as a normalized vector of 128 elements [6].

\subsection{SURF}

SURF (Speeded up Robust Features) has been recently published by Bay et al. (2006). Like SIFT, the SURF approach describes a keypoint detector and descriptor. Keypoints are found by using a so called Fast-Hessian Detector that bases on an approximation of the Hessian matrix for a given image point. The responses to Haar wavelets are used for orientation assignment, before the keypoint descriptor is formed from the wavelet responses in a certain surrounding of the keypoint. The descriptor vector has a length of 64 floating point [7].

\subsection{BRIEF}

BRIEF (Binary Robust Invariant Elementary Features) includes only descriptor. It has been published by Calonder et al. (2010). BRIEF is binary descriptor, it describes image patches that could be effectively classified on the basis of the small number of pair-wise intensity comparisons. Binary descriptors are very fast and outperform SIFT and SURF in speed. BRIEF choose random sampling pairs depending on sampling geometries. Five different approaches to choosing the test locations were presented. Then comparing between two intensity values is done. If the first value is bigger than the second value in final vector will be written 1 otherwise 0 . Final descriptor has length 128/256/512 bits depending on pair count [8].

\subsection{ORB}

ORB (Oriented FAST and Rotated BRIEF) contains both detector and descriptor. It was published by Ruble et al. (2011). ORB detector is based on FAST detector. However, it is called OFAST and and it is rotation invariant modification of FAST. ORB detector takes one parameter, the intensity threshold between the center pixel and those in circular ring about the center. For orientation, compensation is used corner orientation method called intensity centroid. ORB descriptor is based on BRIEF method. They used rBRIEF which is basically improved modification of BRIEF. To recover from the loss of variance in steered BRIEF and to reduce correlation among the binary test they used learning method for choosing a good subset of binary tests. The result is called rBRIEF [9].

\subsection{BRISK}

BRISK (Binary Robust Invariant Scalable Keypoints) as published by Leutenegger et al. (2011) includes both detector and descriptor. Detector is inspired by AGAST [12] method which is a modification of FAST. The keypoints are identified across both the image and scale dimensions using saliency criterion. Keypoints are detected in octave layers of image pyramid as well as in layers inbetween. The location and the scale of keypoint are obtained in continuous domain via quadratic function fitting. Descriptor is different from descriptors BRIEF and ORB. BRISK use sampling pattern it is composed out of concentric rings. On each sampling point is taken a small patch around it and is applied Gaussian smoothing. The size of the deviation of Gaussian filter is defined by size of circle around each sample point. From this pattern long and short distance pairs was defined. Long pairs are used to determine orientation. BRISK uses local gradients between the sampling pairs for computing the orientation. Short pairs are used for intensity comparisons for final descriptor creation [10]. 


\subsection{FREAK}

FREAK (Fast Retina Keypoint) is binary descriptor published by Alahi et al. (2012). FREAK is similar to BRISK by having a sampling pattern and orientation compensation mechanism and also to ORB by using machine learning techniques to learn the best set of sampling pairs. FREAK has sampling pattern inspired by retina in human eye. It uses the retinal sampling grid which is circular but it has higher density of points near the center. Sampling grid corresponds with the distribution of the receptive fields over the retina. Each sampling point is smoothed by Gaussian kernel. Radius of the circle illustrates the size of deviation of the kernel. FREAK tries to learn the pairs by maximizing variance of the pairs and taking pairs that are not correlated. Orientation compensation is similar to BRISK but instead of using long distance pairs FREAK uses a predefined set of 45 symmetric sampling pairs. Final descriptor is created by using four clusters of pairs. Each cluster is composed from 128 sampling pairs. That means final descriptor have 512 bits. The first cluster involves mainly peripheral receptive fields whereas the last ones implicate highly centered fields. Matching steps takes advantage of the coarse-to-fine structured of FREAK descriptor [11].

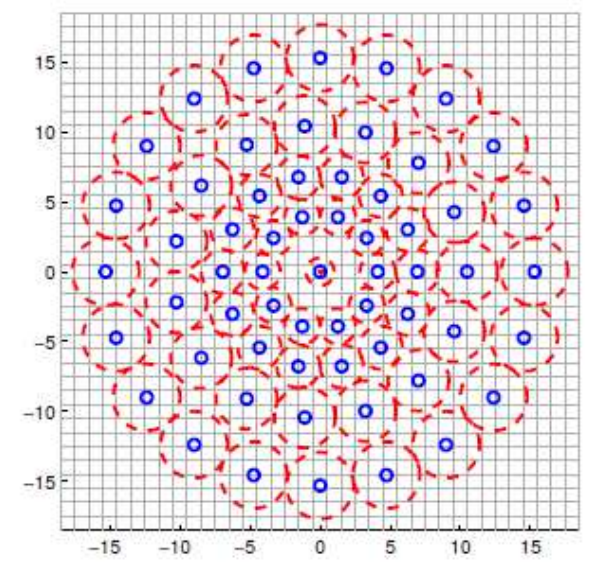

a) BRISK sample pattern

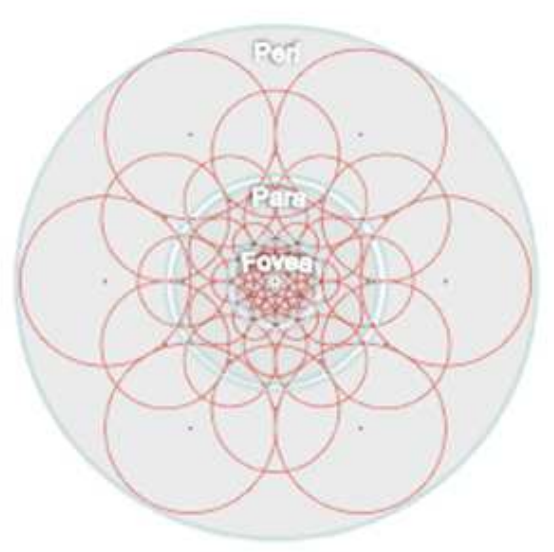

b) FREAK sample pattern

Fig. 4: Binary descriptor sample pattern.

\section{Experiments and results}

In this section we evaluate the results of the tested algorithms for local feature extraction on wildlife mammal samples in infrared domain. All methods were implemented in $\mathrm{C}_{++}$using OpenCV library.

\subsection{Mammal database}

Wildlife mammal database has not been created yet. Thus, mammal database was created. It was created from trail cameras photos. Mammal samples were handcrafted from photos. The dataset has 5 kinds of mammals: wolf, bear, deer, fox, and boar. Each class has 50 different images of animals totalling 250 images. Images for each class are different. There are some variances depending on image size, animal poses, amount of noise, illumination distribution etc. Samples of each class are illustrated on Figure 5, there were shown differences in each class. As we can see, fur of each kind of animals reflects various amount of infrared light. Also it depends on distance between light source and animal. Very helpful information in our future work is the fact that a lot of wild mammals have reflective layers in their eyes. Therefore, animals captured in near infrared domain have shinning eyes which can be easily detected and could be helpful in animal detection process. 


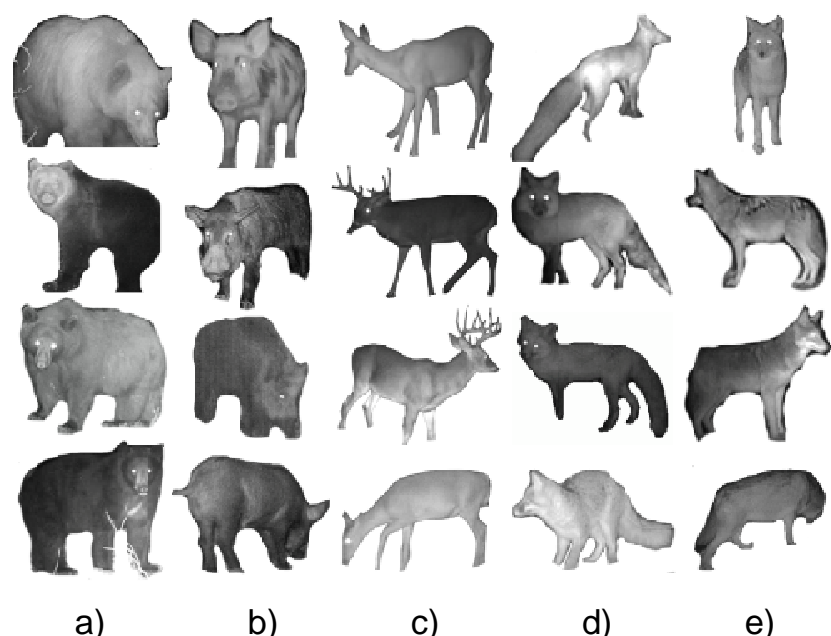

Fig. 5: Illustration of 5 kinds of animals from mammal database: a) bear, b) boar, c) deer, d) fox, e) wolf.

\subsection{Testing process}

At first we do not want to find keypoints on white areas around the animals. Thus binary masks were created for each image. Mask was based on thresholding. It helps define area where keypoint extraction could be done. Next keypoint extraction is done. For keypoint extraction was used SIFT, SURF, ORB, BRISK methods. Then keypoints filtering were applied to select only the strongest keypoints from image. After that description method were applied on each image. For keypoints description were used SIFT, SURF, ORB, BRISK and FREAK methods. The set of descriptors was created from whole animal database. This dataset was divided into two sets for training and for testing.

Two methods for descriptor extraction were used. Method 1 (KPM1) extract from each image keypoints and also immediately descriptors was created. Thus each class had a different amount of descriptors. Method 2 (KPM2) at first extract keypoints from all images from same class. Then specified number of keypoints was used for feature extraction. So each class has the same amount of descriptors.

For classification Brute Force matcher were used. Each descriptor from one test image was matched with all descriptors from all train images. For matching was used Euclidean distance between descriptors. This process returned the number of matched descriptors between images. When all matches were calculated we also used two methods for result classification. First method 1 (MM1) return the train image class with maximum count of matched descriptors with descriptors from tested image. The method 2 (MM2) calculate amount of matches for whole class. Then the train image is classified as class with most matches. The process of feature extraction and classification are illustrated on the Figure 6.

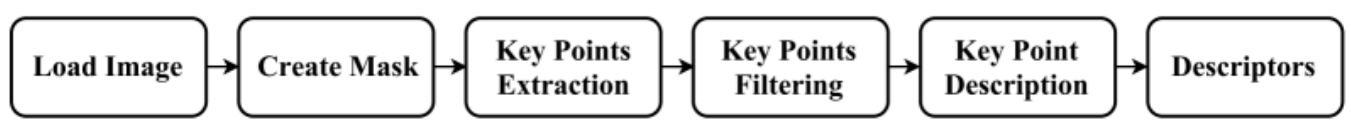

a) Local feature extraction

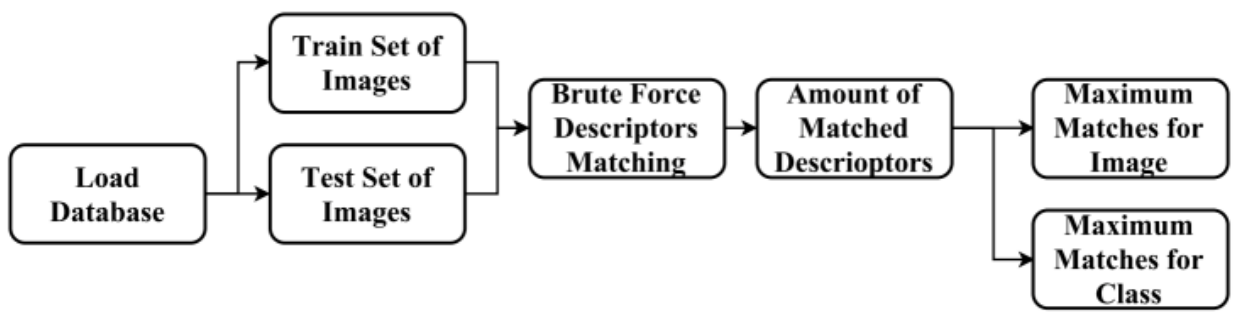

b) Descriptors matching process

Fig. 6: Illustration of: a) local feature extraction process, b) descriptors matching process. 


\subsection{Results}

Test set contains twenty images from each class so for training were used thirty images for one class. That mean one hundred images were tested opposite to one hundred and fifty train images from five classes. Thirty two tests were realized for each combination of keypoint and matching methods. Figure 5 shows the best results for each feature extraction method. Also process time was saved.

The best results were reached with SURF detector and descriptor with $67 \%$ accuracy. Also all 20 deer were classified correctly. On the other side only eight samples of wolves were correctly classified as wolf. Most of wolves were classified as fox or boar. Also processing time is very good opposite to other methods. For example BRISK detector and descriptor reach only $32 \%$ accuracy with the worst processing time almost 853 seconds. Some of methods have zero accuracy for some classes. BRISK did not recognize any samples from classes: boar, fox, and wolf. And also SIFT, ORB and combination of SURF detector with FREAK descriptor. All methods have problem with wolf classification due to similarity with foxes and boars. Maximum accuracy for wolves was $40 \%$. The first keypoint extraction method was better for most of feature extraction methods. And for matching were better the second method. Also a lot of parameters influence the final result.

Tab. 2: Experimental results.

\begin{tabular}{|c||c|c|c|c|c|c|c|}
\hline $\begin{array}{c}\text { Method Detector- } \\
\text { Descriptor }\end{array}$ & $\begin{array}{c}\text { SIFT- } \\
\text { SIFT }\end{array}$ & $\begin{array}{c}\text { SIFT- } \\
\text { SURF }\end{array}$ & $\begin{array}{c}\text { SURF- } \\
\text { SIFT }\end{array}$ & $\begin{array}{c}\text { SURF- } \\
\text { SURF }\end{array}$ & $\begin{array}{c}\text { ORB- } \\
\text { ORB }\end{array}$ & $\begin{array}{c}\text { BRSIK- } \\
\text { BRISK }\end{array}$ & $\begin{array}{c}\text { SURF- } \\
\text { FREAK }\end{array}$ \\
\hline \hline $\begin{array}{c}\text { KeyPoint } \\
\text { extraction method }\end{array}$ & KPM1 & KPM1 & KPM1 & KPM1 & KPM2 & KPM1 & KPM2 \\
\hline Matching method & MM1 & MM1 & MM2 & MM2 & MM2 & MM2 & MM2 \\
\hline Bear & $60,00 \%$ & $40,00 \%$ & $70,00 \%$ & $75,00 \%$ & $50,00 \%$ & $\mathbf{9 5 , 0 0 \%}$ & $75,00 \%$ \\
\hline Boar & $40,00 \%$ & $\mathbf{7 0 , 0 0} \%$ & $0,00 \%$ & $60,00 \%$ & $60,00 \%$ & $0,00 \%$ & $40,00 \%$ \\
\hline Deer & $75,00 \%$ & $90,00 \%$ & $95,00 \%$ & $\mathbf{1 0 0 , 0 0} \%$ & $90,00 \%$ & $65,00 \%$ & $60,00 \%$ \\
\hline Fox & $0,00 \%$ & $5,00 \%$ & $70,00 \%$ & $60,00 \%$ & $0,00 \%$ & $0,00 \%$ & $\mathbf{7 5 , 0 0 \%}$ \\
\hline Wolf & $25,00 \%$ & $20,00 \%$ & $35,00 \%$ & $\mathbf{4 0 , 0 0} \%$ & $5,00 \%$ & $0,00 \%$ & $0,00 \%$ \\
\hline Overall accuracy & $\mathbf{4 0 , 0 0 \%}$ & $\mathbf{4 5 , 0 0} \%$ & $\mathbf{5 4 , 0 0} \%$ & $\mathbf{6 7 , 0 0} \%$ & $\mathbf{4 1 , 0 0} \%$ & $\mathbf{3 2 , 0 0} \%$ & $\mathbf{5 0 , 0 0 \%}$ \\
\hline $\begin{array}{c}\text { Overall Processing } \\
\text { Time [s] }\end{array}$ & 309,65 & 193,82 & $\mathbf{4 7 9 , 3 9}$ & 97,42 & $\mathbf{4 8 , 1 9}$ & 852,92 & 144,72 \\
\hline
\end{tabular}

\section{Conclusions}

In this paper some of local feature extraction method was compared for animal classification purpose in near infrared domain images. Animal classification from NIR images is difficult task due to image quality with a lot of noise and a various illumination conditions. We concluded using only local feature method with brute force matcher is not enough for good classification. In future we will try to improve result with using Support Vector Machine learning method and also compare it with Neural Networks. Due to various illumination, some animals closer to camera are white, but it is impossible to find some keypoints in this areas, thus using only local descriptors is not enough, therefore combination with some global features will be used.

\section{Acknowledgement}

This work was supported by project EUREKA no. E!6752 - DETECTGAME: R\&D for Integrated Artificial System for Detecting the Wildlife Migration and project "Centre of excellence for systems and services of intelligent transport ", ITMS: 26220120028, co-funded from EU sources and European Regional Development Fund.

\section{References}

[1] MINISTERSTVO VNÚTRA SLOVENSKEJ REPUBLIKY: Ročenka dopravnej nehodovosti. Available at: http://www.minv.sk/?rocenkaSR. February, 2015 (in Slovak).

[2] ISO 20473:2007. Available at: https://www.iso.org/obp/ui/ \#iso:std:iso:20473:ed-1:v1:en. March, 2015. 
[3] FLIR Systems Inc.: What's the difference between thermal imaging and night vision?. Available at: http://www.flir.com/cvs/americas/en/ view/?id=30052. March, 2015.

[4] DONG-GEN LUO - WENDY W. S. YUE - PETRI ALA-LAURILA - KING-WAI YAU: Activation of Visual Pigments by Light and Heat. In: Science. 2011, Vol. 332, Issue 6035, p. 1307 - 1312. DOI: $10.1126 /$ science.1200172.

[5] MATUSKA, S. - HUDEC, R. - BENCO, M. - KAMENCAY, P. - ZACHARIASOVA, M.: A novel system for automatic detection and classification of animal. In: ELEKTRO. 2014, p.76, 80, p. 19 20.

[6] LOWE, DAVID G.: Object recognition from local scale-invariant features. In: Computer Vision. Vol. 2, ICCV'99 Proceedings of the international conference, p. 1150.

[7] BAY, HERBERT - TINNE TUYTELAARS, - LUC VAN GOOL.: SURF: Speeded up robust features. In: Computer Vision - ECCV. 2006, Vol. 3951, p. 404 - 417. Springer Berlin Heidelberg, 2006.

[8] CALONDER, M. et al.: Brief: Binary robust independent elementary features. In: Computer Vision - ECCV. 2010, Vol. 6314, p. 778 - 792. Springer Berlin Heidelberg, 2010.

[9] RUBLEE, E. et al.: ORB: an efficient alternative to SIFT or SURF. In: ICCV'11 Proceedings of the 2011 International Conference on Computer Vision, p. 2564 - 2571.

[10] LEUTENEGGER, S. - CHLI, M. - SIEGWART, R. Y.: BRISK: Binary Robust invariant scalable keypoints. In: Computer Vision (ICCV). 2011 IEEE International Conference on, Barcelona, 2011, p. 2548 - 2555. DOI: 10.1109/ICCV.2011.6126542.

[11] ALAHI, A. - ORTIZ, R. - VANDERGHEYNST, P: FREAK: Fast Retina Keypoint. In: Computer Vision and Pattern Recognition (CVPR). 2012 IEEE Conference on, Providence, RI, 2012, p. 510 517. DOI: 10.1109/CVPR.2012.6247715.

[12] MAIR, E. - HAGER, G.D. - BURSCHKA, D. - SUPPA, M. - HIRZINGER, G.: Adaptive and generic corner detection based on the accelerated segment test. In: Proceedings of the European Conference on Computer Vision (ECCV'10), September 2010.

[13]ROSTEN, E. - DRUMMOND, T.: Machine learning for high speed corner detection. In: 9th European Conference on Computer Vision, Vol. 1, 2006, p. 430 - 443.

[14] LOWE, D.G.: Distinctive Image Features from Scale-Invariant Keypoints. In: International Journal of Computer Vision. 2004, Vol. 60, Issue 2, p. 91 - 110. DOI:10.1023/B: VISI. 0000029664. 99615.94. 